\title{
Fast neutron source detection at long distances using double-scatter spectrometry
}

\author{
L. Forman and Peter E. Vanier, Brookhaven National Laboratory \\ and Keith Welsh, State University of New York at Stony Brook
}

\begin{abstract}
Fast neutrons can be detected with relatively high efficiency, $>15 \%$, using two planes of hydrogenous scintillator detectors where a scatter in the first plane creates a start pulse and scatter in the second plane is separated by time-offlight. Indeed, the neutron spectrum of the source can be determined as the sum of energy deposited by pulse height in the first added to the energy of the second found by time-of-flight to the second detector. Gamma rays can also create a double scatter by Compton interaction in the first with detection in the second, but these events occur in a single time window because the scattered photons all travel at the speed of light. Thus, gamma ray events can be separated from neutrons by the time-of-flight differences. We have studied this detection system with a Cf-252 source using Bicron $501 \mathrm{~A}$ organic scintillators and report on the ability to efficiently detect fast neutrons with high neutron/gamma detection ratios.

We have further studied cosmic-ray neutron background detection response that is the dominant background in long range detection. We have found that most of the neutrons are excluded from the time-of-flight window because they are either too high in energy, $>10 \mathrm{MeV}$, or too low, $<10 \mathrm{keV}$. Moreover, if the detection planes are position-sensitive, the angular direction of the source can be determined by the ratio of the energy of scattered protons in the first detector relative to the position and energy of the scattered neutron detected in the second. This ability to locate the source in theta is useful, but more importantly increases the signal to noise relative to cosmic-ray produced neutrons that are relatively isotropic. This technique may be used in large arrays to detect neutrons at ranges up to 0.5 kilometer.
\end{abstract}

Key words: fast neutron; time-of-flight; organic scintillator; cosmic ray; plutonium.

\section{INTRODUCTION}

In principle, fast neutrons can be used to detect plutonium at long distances because the air scattering mean free path is long ( $>100$ meters for $2 \mathrm{MeV}$ neutrons) and the background, generally related to cosmic-ray reactions, is small. Since neutron detectors can be made to be very large, the fundamental detection limit is usually the neutron production by cosmic-ray interactions. Reduction of background can be accomplished in two ways. First, although fast neutron spectra from plutonium are similar to the spallation portion of the cosmic neutron spectrum, the total spectra are not the same and this difference may be used separate the phenomena. Second, source neutrons radiate from a single direction, whereas cosmic-ray generated neutrons are reasonably isotropic. Therefore, a directional detector can improve the signal-to-noise ratio. The neutron double-scatter spectrometer measures neutron spectra and does so directionally. In this paper we will present data from Cf-252 that demonstrate that it can be used in the neutron energy region 0.1 $6.0 \mathrm{MeV}$ which is appropriate for detection of plutonium neutron spectra.

The neutron double-scatter spectrometer relies on a proton scatter in the first plane of the detector, creating a start pulse with energy determined by pulse height, followed by a scatter in the second plane, where the energy of the scattered neutron can be determined by time-of-flight, as depicted in Figure 1 . This method was first presented by Preszler ${ }^{1,2}$ as a method for study of thermonuclear temperature broadening of the DD (2.5 MeV) and DT (14 MeV) neutrons from plasma reactions and has been used for this purpose by others ${ }^{2}$. Measurements of thermonuclear emission peaks are often limited to a narrow energy region and this is a first application to the relatively low energy spectra of Cf-252 and $\mathrm{Pu}-240$. 


\section{EXPERIMENTAL METHODS}

Consider the geometry we have used for most of our measurements where the source is aligned with the spectrometer axis, $\theta=0$, and the distance between the source and first detector, $R$, is greater than its diameter, $2 r_{1}$. For a neutron striking the center of detector 1 and scattering isotropically in the center of mass system, the maximum possible scattering angle is given by $\left.\sin \phi_{\max }=\mathrm{r}_{2} /\left(\mathrm{L}^{2}+\mathrm{r}_{2}\right)^{2}\right)^{0.5}$, where $\mathrm{r}_{2}$ is the radius of detector 2 , and the geometric probability of scattering in the first reaching the second can be shown to be $\mathrm{r}_{2}{ }^{2} /\left(\mathrm{L}^{2}+\mathrm{r}_{2}{ }^{2}\right)$.

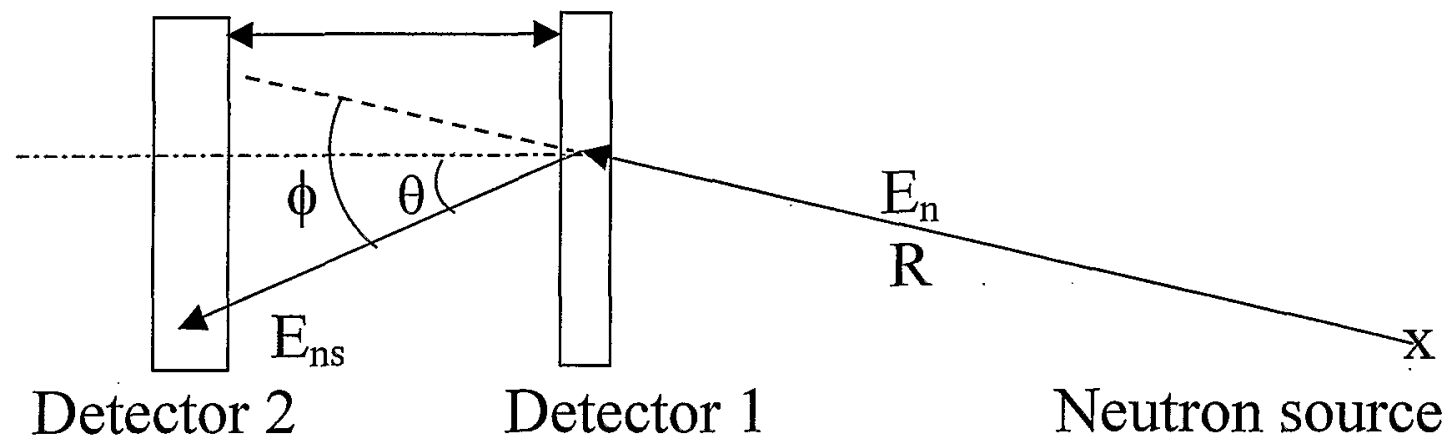

Figure 1: Neutron double scatter geometry.

A neutron originating from a source with energy $E_{n}(\mathrm{MeV})$, scatters off a proton in detector 1 in the direction $\phi$. The scattered neutron, with energy $E_{n s}$ may then be detected in the second detector after having traveled a distance $L / \cos \theta$, where $\mathrm{L}$ is the distance in $\mathrm{cm}$ between detectors with associated time-of-flight in $\mathrm{ns}$, TOF, and $\theta$ is the scatter angle relative to the axis. The kinematic equations describing the neutron scattering are:

$$
\begin{aligned}
& \mathrm{E}_{\mathrm{n}}=\mathrm{E}_{\mathrm{ns}}+\mathrm{E}_{\mathrm{p}} \\
& \mathrm{E}_{\mathrm{ns}}=\left(\frac{0.723 \mathrm{~L}}{T O F \cos \theta}\right)^{2} \\
& \tan ^{2} \phi=\frac{\mathrm{E}_{\mathrm{p}}}{\mathrm{E}_{\mathrm{ns}}}
\end{aligned}
$$

Finally, the probability that a neutron will undergo a double scatter event, $P\left(E_{n}\right) \mathrm{dE}_{\mathrm{n}}$ :

$$
\mathrm{P}\left(\mathrm{E}_{\mathrm{n}}\right) d E_{\mathrm{n}}=\mathrm{S}\left(\mathrm{E}_{\mathrm{n}}\right) \mathrm{P}_{\mathrm{n} 1}\left(\mathrm{E}_{\mathrm{n}}\right) \frac{\pi r_{1}^{2}}{4 \pi R^{2}} \mathrm{P}_{\mathrm{n} 2}\left(\mathrm{E}_{\mathrm{n} S}\right) \frac{r_{2}^{2}}{\left(L^{2}+r_{2}{ }^{2}\right)} d E_{n}
$$

$P_{n 1}\left(E_{n}\right)$ is the probability that a neutron will scatter in the first plane, and $P_{n 2}\left(E_{n s}\right)$ is the probability that the scattered neutron will escape the first and interact in the second. For the thicknesses of detectors we used, 2.5 and $5.1 \mathrm{~cm}$ respectively, probabilities of interaction at $2 \mathrm{MeV}$ are 0.28 and 0.48 , respectively. For large diameter detectors, $r_{1}$ and $r_{2}$ can be greater than $L$ and therefore the spectrometer can be quite efficient. 


\section{COSMIC-RAY BACKGROUND}

NASA Langley Research Center has supported a comprehensive study of radiation exposures from atmospheric ionizing radiation (AIR). P. Goldhagen and his collaborators have published $d^{4,5}$ the cosmic-ray produced neutron spectra portion of this study. This experimental data is quite helpful for understanding the background environment of neutron detectors. Galactic cosmic rays are energetic charged particles that originate outside the solar system. Cosmic-ray neutron production will vary with latitude (the earth's magnetic field deflects cosmic rays), altitude (the atmosphere attenuates cosmic rays and neutrons), and other factors such as solar activity which affect the magnetic field in the solar system. Although there are many interaction possibilities, the most important are direct reactions that produce $10-1,000$ $\mathrm{MeV}$ neutrons, and spallation reactions that generate $0.1-10 \mathrm{MeV}$ neutrons, see Figure 2. Neutrons in the $10^{-8}-10^{-2}$ $\mathrm{MeV}$ range are indicative of neutrons slowing down in air (equilibrium slowing-down spectra). At the low energy extreme, thermal neutrons, $2.5 \times 10^{-2} \mathrm{eV}$, are measured at sea level after thermalization, predominantly occurring in the ground. Finally, there is structure in the higher energy range that correlates with resonance scattering of air nuclei.

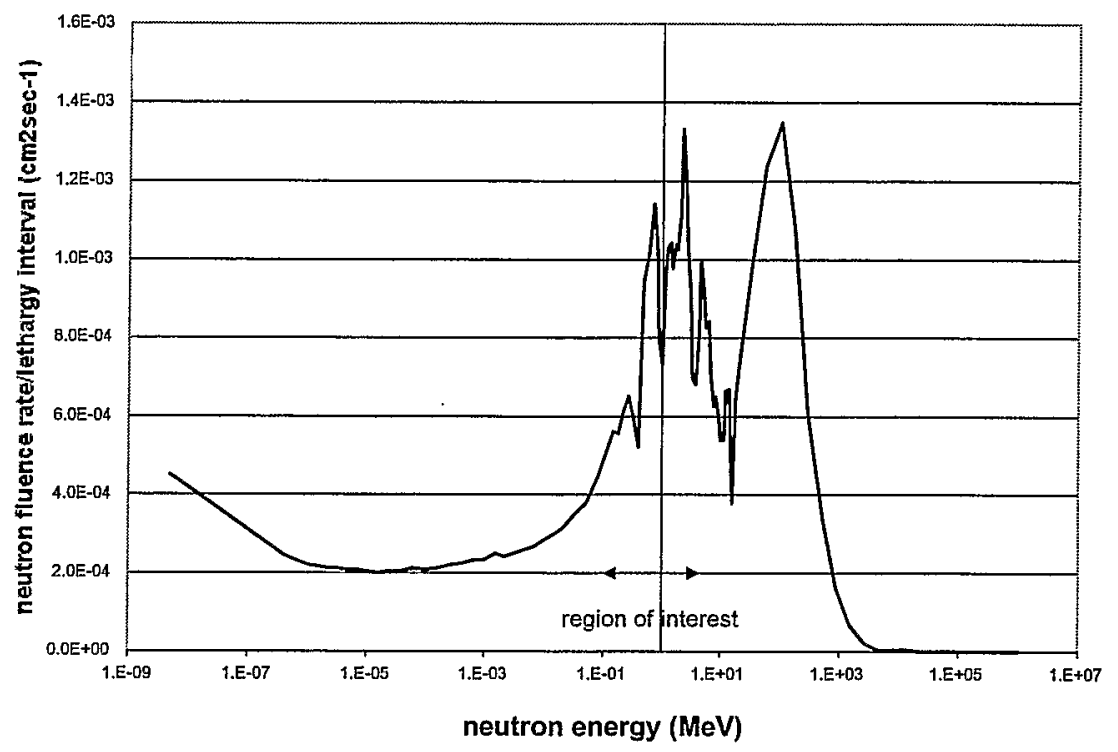

Figure 2: Cosmic ray neutron spectra at sea level (Goldhagen et. al. in Reference 4). The spectra have been plotted in the vertical scale in units of $\mathrm{E} d \phi / \mathrm{dE}$ which visually represents the area. The operating region of the double scatter spectrometer is $0.1-6 \mathrm{MeV}$ including less than one third of the total cosmic ray background.

\section{DETECTOR RESPONSE}

Experiments were carried out using Bicron type 501A scintillators $5 \mathrm{~cm}$ diameter, $2.5 \mathrm{~cm}$ thick, for the first detector and $13 \mathrm{~cm}$ diameter, $5 \mathrm{~cm}$ thick, for the second. Detector pulses were routed through constant fraction discriminators to a time-to-amplitude converter, and spectra recorded in a multichannel analyzer. Since these detectors react to both neutrons and photons, events in the time region of interest are as follows:

1. Neutron double scatter

2. Photon double scatter (Compton event in first detector)

3. Fission gamma ray generated start pulse followed by neutron detection in the other detector.

4. Random photon background.

Other processes may occur that can produce events on this time frame, such as two separate neutrons from one spontaneous fission event, each registering in the separate detectors, but these are less likely. 


\section{NEUTRON DOUBLE-SCATTER SPECTROMETER RESPONSE MEASUREMENT}

The spectra shown in Figure 3 are the time differences of events relative to the start time in detector 1. Sources were located $18 \mathrm{~cm}$ from and perpendicular to the first detector. A delay in the signal line of the second detector causes the timing signal to be registered at about $45 \mathrm{~ns}$ when the detector signals start at the same time, or nearly the same time for gamma/gamma double scatter. If the second detector fires first, the time between pulses is less than $45 \mathrm{~ns}$. Cf- 252 emits fission gamma rays and neutrons that are correlated in time, and photons from other reactions, such as radioactive decay of fission products, that are not correlated with neutron emission. Note that the detector responses are normalized to the peak, or gamma/gamma coincidence:

1. The gamma/gamma peak has about the same half-width for the Cs-137 and Cf-252 sources. This time width is relatively independent of the spacing between detectors.

2. Within 5 nanoseconds of the peak, the Cs- 137 signal becomes negligible relative to the $\mathrm{Cf}-252$ neutron signal.

3. The shapes of the double-scatter Cf-252 timing spectra, shown to the right of the gamma/gamma peak, are quite different because the flight path of scattered neutrons between detectors is either $8 \mathrm{~cm}$ or $45 \mathrm{~cm}$.

4. The neutron double-scatter signal to gamma-ray background ratio is greater for the detectors separated by $45 \mathrm{~cm}$ than $8 \mathrm{~cm}$. In general, this is explained by the wider dispersion by neutrons at the longer flight path.

5. Coincidence events to the left of the gamma/gamma peak primarily are from fission gamma rays creating a start pulse in the second detector, g2, followed by neutron time of flight detection from the source to the first detector, $\mathrm{n} 1$. The spectral response function of the detector used in this mode, $\mathrm{g} 2 \mathrm{n} 1$, is different from the neutron double scatter mode. For example, the g2n1 mode detection efficiency is proportional to the product of source solid angles of both detectors whereas the double scatter efficiency is proportional only to the source solid angle of the first detector.

6. The shapes of g2n1 timing spectra in Figure 2 are relatively independent of detector separation. This is because the source distance to the first detector is the same, $18 \mathrm{~cm}$, for both measurements, and the flight path for time-of-flight dispersion (gamma ray flight times) difference caused by the difference in the position of the second detector is less than a nanosecond.

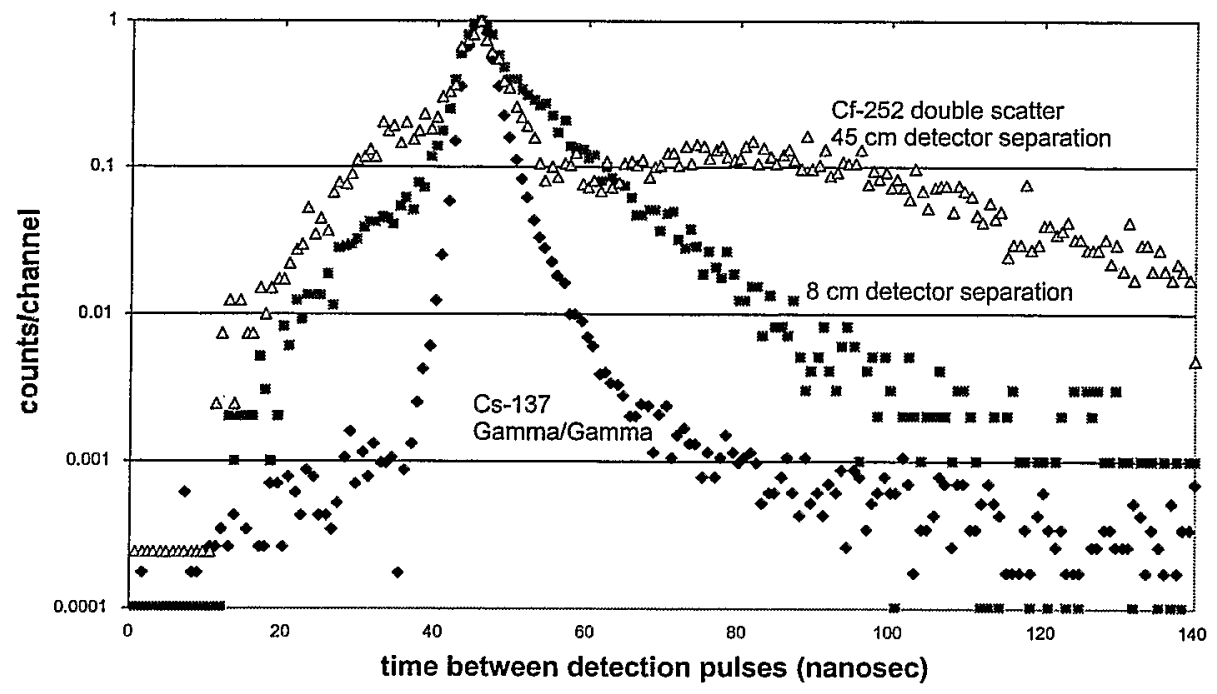

Figure 3: The spectrometer response to Cf-252 spontaneous fission and Cs-137 sources. 


\section{DIRECTIONAL RESPONSE}

With the source positioned at $90^{\circ}$ to the detector planes, there is expected to be little probability of a neutron double scatter relative to the chance of a fission gamma ray creating a start pulse in either detector followed by a neutron pulse in the other. The neutron time-of-flight signal is therefore expected to be reasonably symmetric as is observed (Fig. 4). When the distance from the source increases, the neutron time-of-flight path becomes longer and the signal becomes wider and lower, as observed.

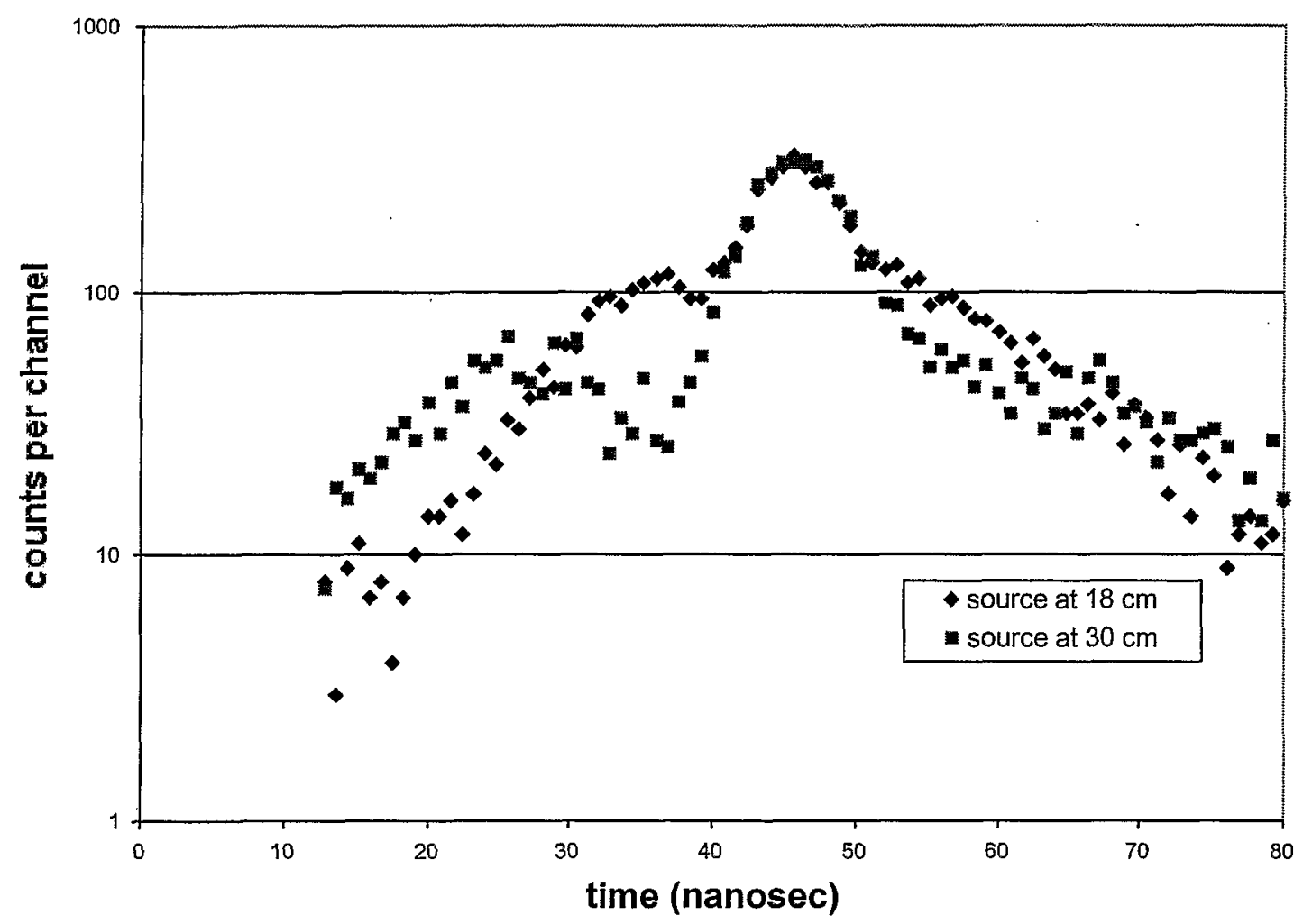

Figure 4: Spectrometer response to a Cf-252 source with source positioned at $90^{\circ}$

When the source was positioned at the rear of the detector $\left(180^{\circ}\right)$ the device response was again asymmetric, showing a mirror image of the data obtained at $0^{\circ}$, though less sensitive by an order of magnitude relative to the signal from the source placed in the forward direction. This is consistent with the expected shape of the neutron double-scatter distribution (for $180^{\circ}$ the second detector fires first). The differences in size and sensitivity of the two detector planes are sufficient to explain the marked change in sensitivity from the forward to the reverse direction.

In summary, the spectrometer coincidence time spectra are theoretically related to the direction of the source for the gross angles of $0^{\circ}, 90^{\circ}$, and $180^{\circ}$. We estimate that with most spectrometer geometries, it is possible to compute the angular direction of the source with a resolution of $\pi / 6$. 


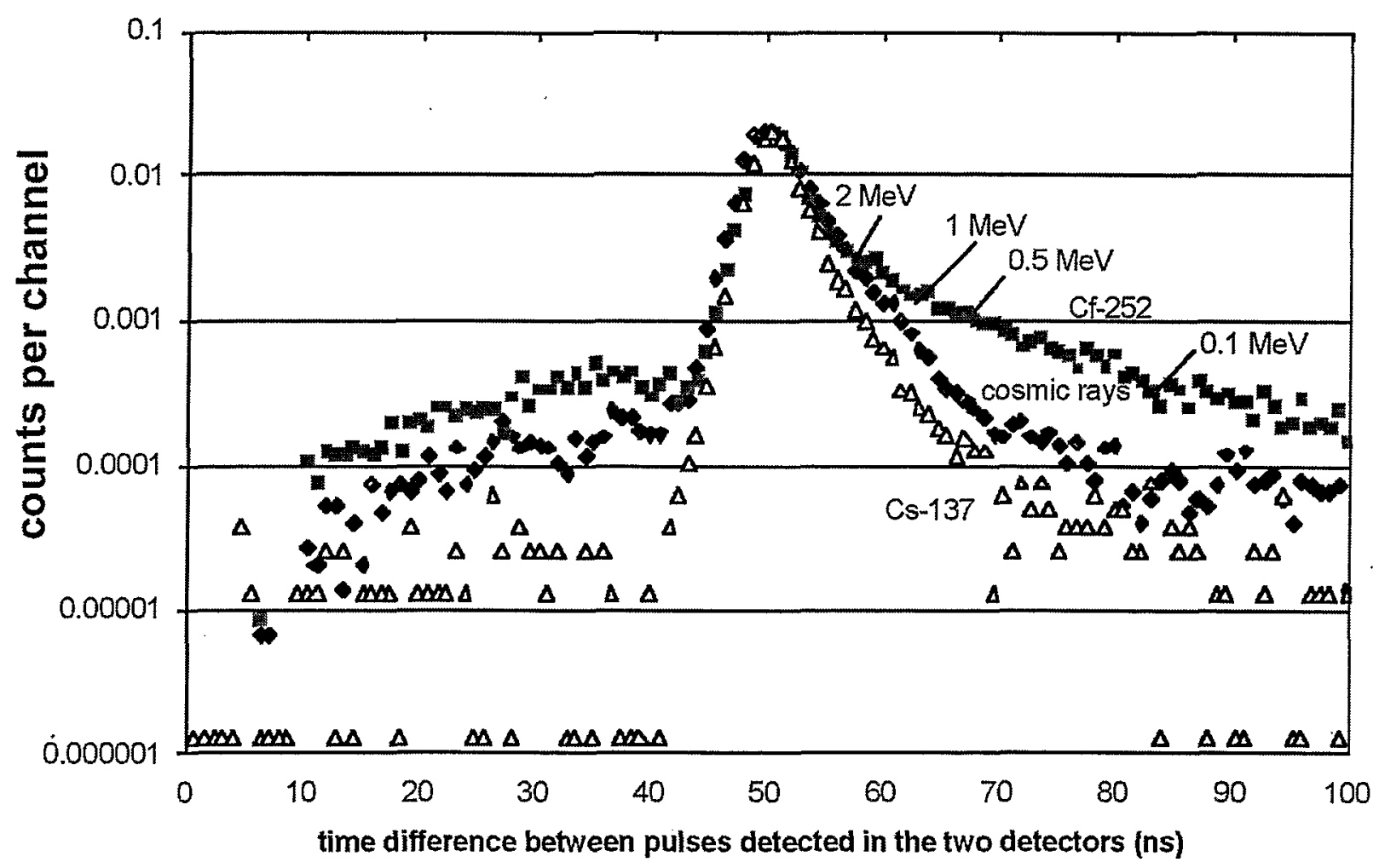

Figure 5: Data acquired with the detection planes $15 \mathrm{~cm}$ apart using the $\mathrm{Cf}-252$ source at $30 \mathrm{~cm}$ normal to detector 1 (squares), and cosmic ray background (diamonds). Also shown are gamma/gamma Cs-137 time spectra (triangles).

From these data, which are normalized to the gamma/gamma peak of the cosmic rays, we make the following observations.

1. The double scatter spectrum of Cf-252 is shown with energy of the scattered neutrons calculated by timeof-flight.

2. The largest difference between the Cf-252 and cosmic ray double scatter spectra is in the energy range 0.1$2 \mathrm{MeV}$. When assuming that the Cs- 137 data are background, the net counts difference between the Cf252 data approach an order of magnitude higher than the cosmic rays in the energy range of interest.

3. Below $0.1 \mathrm{MeV}$, the cosmic ray background begins to approach the $\mathrm{Cf}-252$ data because of the higher content of lower energy neutrons.

4. The double scatter of cosmic ray particles from the backward direction, second detector fires first, is lower than the forward because the second detector is relatively thick to neutrons, about $50 \%$ interaction probability at $2 \mathrm{MeV}$. Thus, lower neutron energies after scatter have a low probability of escaping the scintillator. .

5. The g2n1 time spectra differ in shape from Figure 2 because the neutron time-of-flight path between the source and first detector $(30 \mathrm{~cm})$ is greater than that used in the prior data, $18 \mathrm{~cm}$.

We have not used pulse height analysis in the first detector to determine first scatter energy loss to correlate it with direction; however, in this experimental arrangement the energy loss in the first detector is small. 


\section{ANALYSIS}

The proposed use of this instrument is the detection of neutron sources at long distances. The important technical issues are 1) do the measurements support the double scatter principle in lieu of all other physical processes that can cause coincident pulses? 2) Over what energy range is the instrument useful? 3) How effective is the system in determining direction over the energy region of interest? 4) What neutron detection efficiency can be expected with this and larger detector geometries?

Analysis of the Cf-252 data in Figure 3 is given below.

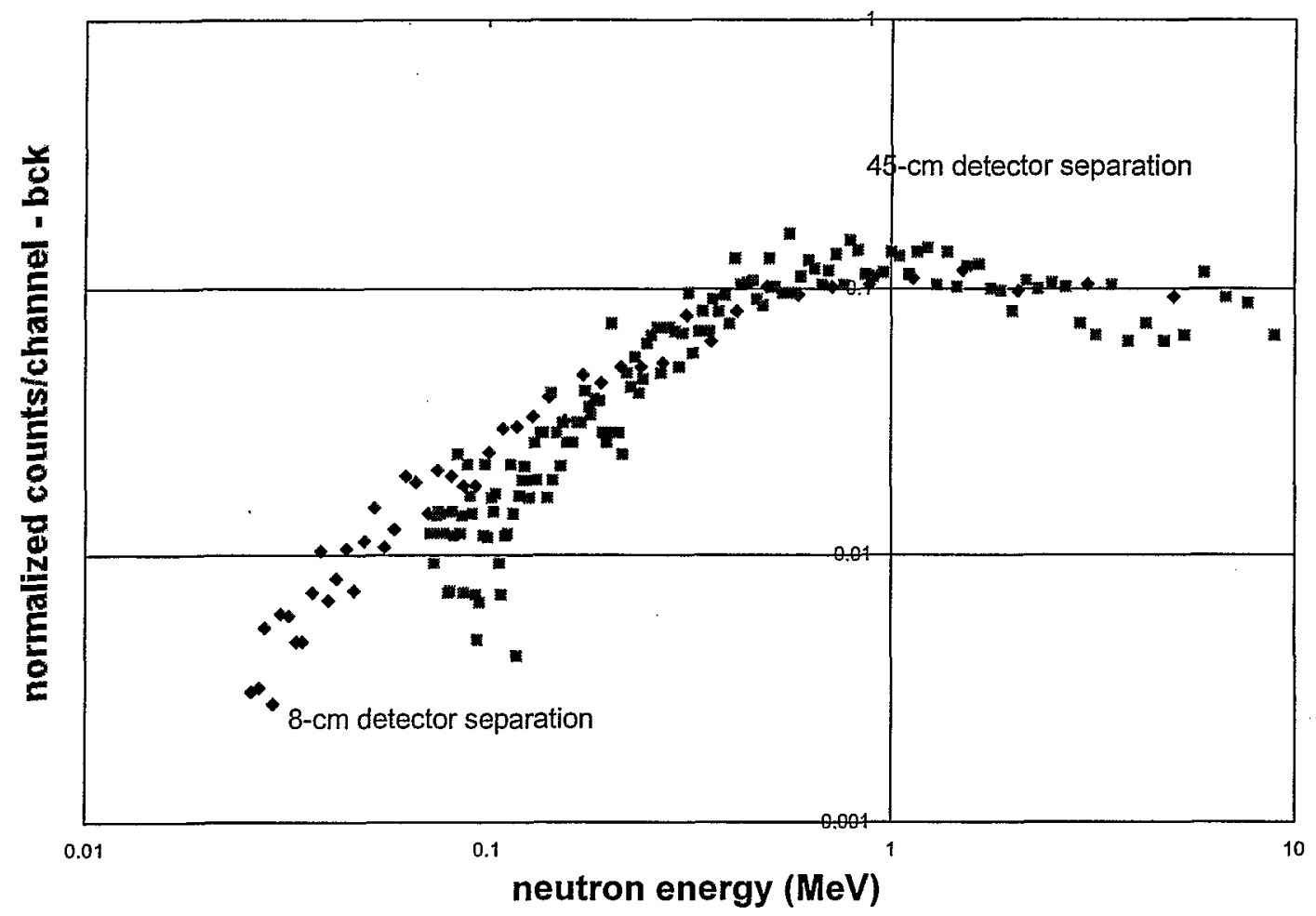

Figure 6: Time of flight data from Figure 3 converted to energy.

Although we have not used Monte Carlo calculations to determine absolute detection response, our first order calculations indicate that the data are consistent with the known Cf-252 spectrum. The spectra indicate that useful data can be obtained between 0.1-6 MeV. The data may also show that there are contributions from the process of a fission gamma ray firing the first detector, g1, followed by a neutron detected in the second, $\mathrm{n} 2$. The neutron flight path for double scatter is the distance between detectors, 8 or $45 \mathrm{~cm}$, whereas with the source at $18 \mathrm{~cm}$ from the first detector, neutron g1n2 time-of-flight paths are 26 or $63 \mathrm{~cm}$. Thus, the g1n2 process would appear in the data as lower energy neutrons more pronounced in the $8 \mathrm{~cm}$ detector separation distance. This would explain why there appear to be a relatively higher number of low energy neutrons in the $8 \mathrm{~cm}$ detector separation data.

We have not determined the energy of the scatter event in the first detector by pulse height analysis to correlate direction from the scattered neutron energy found by time-of-flight of neutron scatter in the second. However, Preszler $^{2}$ was able to separate 12 zenith angles with neutron energies above $1 \mathrm{MeV}$. It is apparent that, in the geometry Preszler studied, there were no provisions to segment the detectors to obtain accurate positions for event occurrence, thus the zenith angle separation could only be resolved by the half angle of divergence between detectors, perhaps $15^{\circ}$. 


\section{CONCLUSIONS}

We have demonstrated that the neutron double scatter spectrometer has a useful operating range of $0.1-6 \mathrm{MeV}$ in which is most of the emission range of spontaneous fission neutron sources, e.g., Pu-240. Recent measurements of cosmic ray related neutron production spectra suggest that this operating range contains less than $33 \%$ of the total background neutrons. The spectrometer can very effectively tell whether a neutron comes from the forward direction and has a resolution of perhaps $\pi / 6$. We have not applied Monte Carlo calculations to model the double scatter spectrometer response and have no definitive predictions of efficiency. However, we estimate that a large detection system could be constructed with greater than 10\% efficiency: a spectrometer system using 3 detection planes might obtain much higher efficiency.

To achieve statistical significance for detection at long range, the magnitude of the cosmic ray background must be reduced. In our approach, the detection planes must be segmented to determine direction of each event with high angular resolution such that the source appears in one angular bin whereas the background is diffuse. If we are able to achieve $10^{\circ}$ angular resolution and are sensitive to $1 / 3$ of the background energy spectra, we could reduce the background response by perhaps a factor of 60 .

\section{ACKNOWLEDGEMENTS}

This genesis of this project was the development of a low energy neutron spectrometer to benchmark a neutron generator designed for brachytherapy treatment of cancerous tumors. Development of the tumor treatment device was accepted as partial fulfillment for the Ph.D. degree in Physics for Dr. Welsh (our co-author) at State University of New York at Stony Brook. For the accelerator development phase of the work, we are grateful to Dr. Chris Jacobsen, Dr. Lawrence E. Reinstein, and Dr. Allen A. Meek of SUNY at Stony Brook. We further recognize the SUNY Nuclear Structure Group for the Tandem Van De Graaf experimental support in characterizing the scintillator material for neutron response, in particular Mr. Richard Loeffer. Dr. John Hartwell of the Idaho National Environmental and Engineering Laboratory gave us invaluable help with unfolding techniques.

The portion of the project reported here was for long range detection of $\mathrm{Pu}$ neutrons that have a spectral shape similar to Cf-252 which was made available to us at Brookhaven National Laboratory (BNL). We wish to thank Dr. Joseph Indusi, Dr. Ralph James, and Dr. Paul Moskowitz at BNL for their support. Dr. Paul Goldhagen at U.S. Department of Homeland Security Environmental Measurements Laboratory provided some of their most recent cosmic-ray related neutron spectra data and gave us important advice. The work has been performed under the auspices of the U.S. Department of Energy Contract No. DE-AC01-98CH10886.

\section{REFERENCES}

1. A. M. Preszler, W. A. Millard, and S. E. Walker, in Proceedings of the Eleventh Symposium on Fusion Energy Nov., 1985, 3 C04.

2. S. E. Walker, A. M. Preszler, and W. A. Millard, Double scatter neutron time-of-flight spectrometer as a plasma diagnostic, Rev. Sci.. Instrum., 57, 1740-1742, Aug., 1986.

3. T. Elevant, P. v. Belle, G. Grosshög, M. Hoek, O. N. Jarvis, M. Olsson, and G. Sadler, The new JET 2.5-MeV neutron Time-of-Flight spectrometer. Rev. Sci.. Instrum., 63, 4586-4588, Oct., 1992.

4. P. Goldhagen, M. Reginato, T. Kniss, J.W. Wilson, R. C. Singleterry, I. W. Jones, amd W. Van Seveninck, Measurement of the Energy Spectrum of cosmic-ray induced neutrons aboard an ER-2 high altitude airplane, Nuclear Instruments and Methods in Physics Research, A 476, 42-51, 2002.

5. P. Goldhagen, Cosmic-ray neutrons on the ground and in the atmosphere, MRS Bulletin, 131-135, Feb., 2003. 